\title{
Vessels Extraction from Retinal Images
}

\author{
H.S. Bhadauria ${ }^{1}$, S.S. Bisht ${ }^{2}$, Annapurna Singh $^{3}$ \\ Department of Computer Science \& Engineering, G.B. Pant Engineering College, Pauri-Garhwal, \\ Uttarakhand-246 194, INDIA
}

\begin{abstract}
The main cause of eye diseases in the working human is Diabetic retinopathy. Eye disease can be prevented if detects early. The extraction of blood vessels from retinal images is an essential and challenging task in medical diagnosis and analysis. This paper describes the effective and efficient extraction of blood vessels from retinal image by using Kirsch's templates. The Kirsch's edge operators detect the edges using eight filters, generated by the compass rotation mechanism. The method is used to automatic detection of landmark features of the fundus, such as the optic disc, fovea and blood vessels.
\end{abstract}

Keywords: - Diabetic retinopathy, Retinal image, Oculist

\section{Introduction}

The extraction of Retinal blood vessel is an essential step for the diagnosis of various eye diseases. Retinal images of humans play a crucial role in the detection and diagnosis of several eye diseases for the oculists [1-2]. Color retinal images are used in mass screen systemic diseases such as Diabetic Retinopathy (DR). The risk of the Diabetic Retinopa-thy increases with age and small eye blood vessels damaged the due to this disease. The information about blood vessels, such as length, width, diameter and branching pattern, can help to diagnose the symptom of diseases [3-4]. The oculists may examine retinal images and give the diagnostic results by searching the possible anomalies like diabetic retinopathy, glaucoma and retinal artery occlusion. With the help of computer aided diagnosis (CAD), the success rate of the treatment of these diseases may increase significantly. If the retinopathy detects in early stage [5-6], the probability of visual loss resulting from diabetic retinopathy can be prevented. The filtering based method which is used for the effective extraction the blood vessels of retinal images are described in [7-8].

In the past various techniques are used to extract the blood vessels from retinal images. One technique not only finds the blood vessels that originate from a set of primary starting point but also finds all branches off of the primary vessels. The second method use Matched Filter (MF) for the extraction of blood vessels. This method will respond not only to vessels but also to non-vessel edges [9]. The third method is the novel hybrid automatic approach for the extraction of retinal image vessels which reduce the weak edges and noise, and finally results the blood vessels extraction. In this paper a method is proposed which uses the Kirsch's templates for the extraction of the blood vessels from retinal images.

The rest of paper is organized into 5 Sections. The Kirsch's edge operators are described in Section 2. Section 3 explains proposed method for the extraction of blood vessels from retinal images. Section 4 shows the results and discussion and finally, the conclusion is given in Section 5.

\section{Kirsch's Template}

Kirsch templates of size $3 \times 3$ are used for the extraction of blood vessels from retinal image Edge detection is a process of identifying the pixel values in order to get frequently and abrupt changes. The generally output of edge detection through Kirsch template is to produce an image containing grey level pixels of value 0 or 255.The value 0 of pixel grey indicates a black pixel and the value 255 indicates a white pixel. Edge information of a particular and target pixel is checked by determining the brightness level of the neighbouring pixels. If there is no major difference in the brightness levels then there is no possibility of edge in the image. The described procedure is most common and fundamental approach among all the available edge detection algorithms such as, Prewitt, Sobel etc. In this paper Kirsch template technique is used for the extraction blood vessels from retinal images. The Kirsch edge detection algorithm uses a single mask of size $3 \times 3$ and rotates it in 45 degree increments through all 8 directions as shown below:

$$
\left[\begin{array}{rrr}
-3 & -3 & 5 \\
-3 & 0 & 5 \\
-3 & -3 & 5
\end{array}\right]\left[\begin{array}{rrr}
-3 & 5 & 5 \\
-3 & 0 & 5 \\
-3 & -3 & -3
\end{array}\right]\left[\begin{array}{ccc}
5 & 5 & 5 \\
-3 & 0 & -3 \\
3 & -3 & -3
\end{array}\right]\left[\begin{array}{rrr}
5 & 5 & -3 \\
5 & 0 & -3 \\
-3 & -3 & -3
\end{array}\right]
$$




$$
\left[\begin{array}{rrr}
5 & -3 & -3 \\
5 & 0 & -3 \\
5 & -3 & -3
\end{array}\right]\left[\begin{array}{lrr}
-3 & -3 & -3 \\
5 & 0 & -3 \\
5 & 5 & -3
\end{array}\right]\left[\begin{array}{rrr}
-3 & -3 & -3 \\
-3 & 0 & -3 \\
5 & 5 & 5
\end{array}\right]\left[\begin{array}{rrr}
-3 & -3 & 5 \\
-3 & 0 & 5 \\
-3 & 5 & 5
\end{array}\right]
$$

Fig. 1 Kirsch's convolution kernels

The edge magnitude of the Kirsch operator is calculated as the maximum magnitude across all direction. The matrix contains the information of a pixel and its neighbours.

The Kirsch algorithm detects direction of the edge as well as an edge. Accordingly, there are eight possible directions south, east, north, west, northeast, southeast, southwest and northwest as shown in figure 1. Out of the several templates the biggest one is considered for the output value and later the edges are extracted. Kirsch template can set and reset the threshold values to obtain most suitable edge of images. Kirsch template works well for images having clear distinction between the foreground and background. Since the retinal blood vessels can be considered as required foreground information from fundus images, Kirsch algorithm can effectively applied.

\section{Proposed Method}

Major steps of the method consist of four steps as edge detection, false edge exclusion, vessel junction restoration, and vessel labeling.

(1) Detection: Apply Kirsch template to the input retinal image to establish a rule in which it checks the condition for edge detection if it finds positive then it can execute condition further .

(2) False edge removal: If condition is no satisfied, then it cannot go ahead.

(3) Vessel junction restoration: Fix broken junctions intro-duced by Kirsch template. At a broken junction, track and find the direction of a vessel. Extend the vessel in the opposite direction for a certain length. If another vessel is found, bridge the gap and restore the vessel junction.

(4) Vessel labeling: A typical vessel is represented by two parallel edges. vessel labeling step fills the interior pixels of a vessel. The challenging task is to differentiate the area within a vessel and the area between two different vessels that are parallel to each other. Literally it is a comparison method which is used effectively.

\section{Results And Discussion}

A general introduction of the challenges and potential of retinal image analysis was presented. Digital retinal imaging playing an increasingly prominent role in the diagnosis and treatment of eye diseases and the extraction of clinically useful information has become important task. For example, retinal vasculature help to define the characteristics and extent of diseases like diabetic retinopathy and glaucoma, aiding their diagnosis and treatment. Therefore, segmentation of these features becomes a key challenge for proper analysis, visualization and quantitative comparison. This has been the main focus of this paper i.e., segmentation of blood vessels from colour retinal images. In this respect the dataset of 10 retinal images is used to evaluate the method. Some of the images were discarded by ophthalmologists prior to the diagnosis. But such images were included in the database to check the robustness of the developed method. Images that suffered from non uniform illumination and poor contrast were subjected to preprocessing, before subjected to segmentation. Color normalization was performed to attenuate color variations in the image by normalizing the colors of the original retinal image against a reference image. In order to correct non uniform illumination and to improve contrast of an image, contrast-limited adaptive histogram equalization was used. For each image in the database fundus mask was detected, that facilitated the detection of vessel pixels within the region of interest. The colored input retinal images are shown in fig.1(a) and fig. 2(a) and all retinal images converted into grey scaled images as shown in fig1(b), fig2(b) and fig3(b). After that all grey scaled images processed by kirsch's templates and it extracts blood vessels through edge detection technique as shown in fig. 1(c), fig. 2(c) and fig. 3(c)

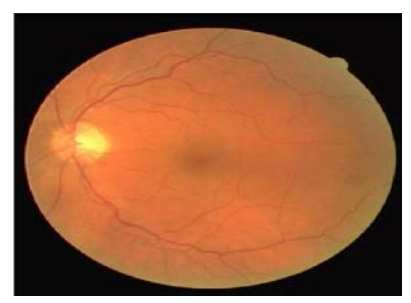

(a) 


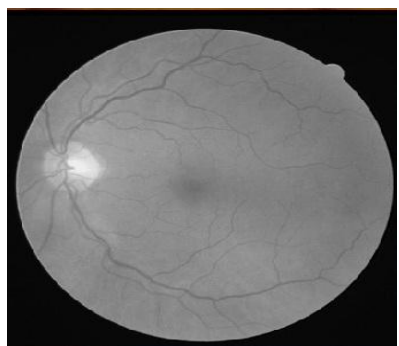

(b)

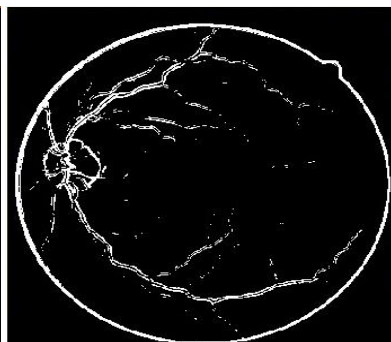

(c)

Fig.1 (a) Color retinal image, (b) Gray retinal image, (c) Extracted blood vessels from retinal image.

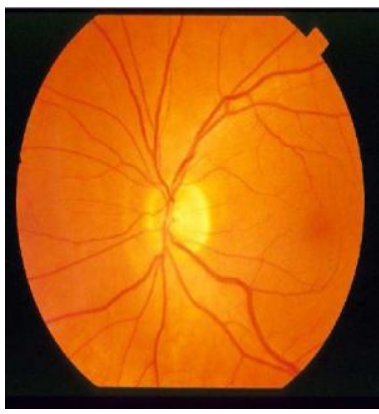

(a)

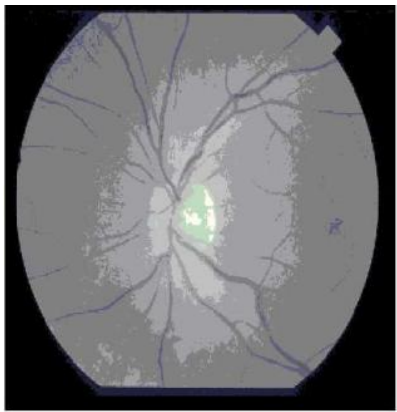

(b)

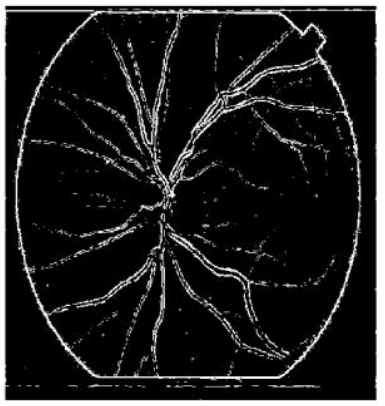

(c)

Fig.2 (a) Color retinal image, (b) Gray retinal image, (c) Extracted blood vessels from retinal image

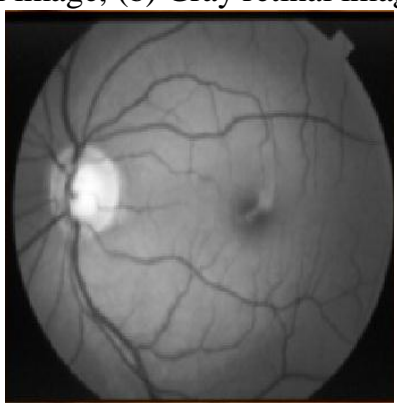

(a)

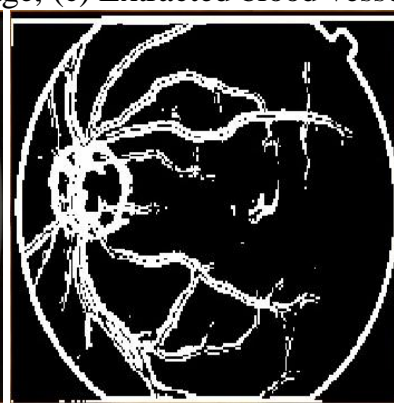

(b)

Fig.2 (a) Gray retinal image, (b) Extracted blood vessels from retinal image

\section{Conclusion}

By above method easily extract efficient retinal blood vessel from retinal image. More than 10 images have been tested and method can extract vessel image successfully. Future work will include improving the presented method and the extraction of other feature such as intensity of image. 


\section{References}

[1]. S. Chaudhuri, S. Chatterjee, N. Katz, M. Nelson, and M. Goldbaum, "Detection of blood vessels in retinal images using two dime nsional matched filters," IEEE Trans. Med. Imaging, vol. 8, March 2008.

[2]. A. Banumathi, R. Karthika, and A. Kumar, "Performance an alysis of matched filter techniques for automated detection of blood v essels in retinal images," Conference on Convergent Technologies for Asia-Pacific Region, vol. 2, pp. 543 - 546, Oct 2003.

[3]. M. Niemeijer, J. Staal, B. van Ginneken, M. Loog, and M. Abramoff, "comparative study of retinal vessel segmentation methods on a new publicly available database,” SPIE Medical Imaging, vol. 5370, pp. 648-656, 2004.

[4]. M. Fraz, M. Javed, and A. Basit, "Evaluation of retinal ve ssel segmen-tation methodologies based on combination of vessel centerlines and morphological processing," IEEE International Conference on Emerging Technologies, oct 2008.

[5]. N. Z. M. of Health, "diabetes in new zealand:models and fo recasts 1996-2011," New Zealand Ministry of Health, Tech. Rep., 2002.

[6]. H. Li and O. Chutatape, "Automatic location of optic disk in retinal images," in IEEE International Conference on Image Processing 2001, august 2001.

[7]. Y. Kwon, A. Bainbridge-Smith, and A. Morris, "Quality as sessment of retinal images," in Proceedings of the Image and Vision Conference New Zealand IVCNZ, p. 281286, Dec 2006.

[8]. H. Li and O.Chutatape, "Automated feature extraction in color retinal images by a model based approach," IEEE Trans. on Medical Imaging, vol. 51, p. 246254, Feb 2004.

[9]. K. W. Tobin, E. Chaum, and V. P. Govindasamy, "Detection o f anatomical structures in human retinal imagery," IEEE Trans. on Medical Imaging, vol. 26, pp. 1729-1739, Dec 2007.

[10]. M. et al, "macula precise localization using digital re tinal angiogra-phies," WSEAS Trans. on Computer Research, vol. 3, pp. 43-50, Jan 2008 . 